Editorial

\title{
Sensor Networks in Structural Health Monitoring: From Theory to Practice
}

\author{
Vasilis Dertimanis *(1) and Eleni Chatzi \\ Department of Civil, Environmental and Geomatic Engineering, ETH Zürich, Stefano-Franscini-Platz 5, \\ 8093 Zürich, Switzerland; chatzi@ibk.baug.ethz.ch \\ * Correspondence: v.derti@ibk.baug.ethz.ch
}

Received: 5 October 2020; Accepted: 12 October 2020; Published: 13 October 2020

The growing attention that structural health monitoring (SHM) has enjoyed in recent years can be attributed, amongst other factors, to the advent of low-cost and easily deployable sensors. The enabling technology has brought forth a new era of structural diagnostic means and is continuously redefining the tools for information processing, data reduction/compression, feature extraction, and smart assessment.

Within the SHM community, novel data-driven or hybrid methods are being developed, demonstrated on field deployments on large-scale systems, including transport, wind energy, and building infrastructure, promoting the actionability of SHM as an essential resource for life-cycle and resilience management. Nonetheless, in optimizing these deployments, a number of open issues remain with respect to the sensing side. These are associated with the type, configuration, and eventual processing of the information acquired from these sensors in order to deliver continuous behavioral signatures of the monitored structures.

Within this context, the aim of this Special Issue is to discuss the latest advances in the field of sensor networks for SHM. The focus lies in both active research on the theoretical foundations of optimally deploying and operating sensor networks, as well as in those technological developments that might designate the next generation of sensing solutions targeted for SHM.

Initiating form the data interpretation side, Argyris et al. [1] introduce a Bayesian framework for finite element model updating using experimental modal data. The novelty lies in utilization of a new likelihood formulation, which enables inclusion of the modal shapes, based on probabilistic treatment of the modal amplitude coherence (MAC) value between the model-predicted and measured mode shapes. The actual case study of the Metsovo bridge is utilized for validation. The modal characteristics of the bridge are identified based on operational modal analysis methods by means of a network of reference and roving sensors. The transitional Markov chain Monte Carlo algorithm is employed for model updating. The method delivers robust uncertainty bounds, outperforming conventional Bayesian formulations, which result in unrealistically thin uncertainty bounds for the model parameters, when a large (redundant) number of sensors is employed.

Moving away from the Bayesian paradigm, Pai et al. [2] describe a framework for practical asset management. In their work, the authors try to relax strong assumptions on uncertainty placed by typical data-interpretation methods, such as Bayesian model updating and residual minimization to better address practical engineering challenges. Error-domain model falsification is compared against these two more established approaches in terms of the ability to provide robust, accurate, engineer-friendly, and computationally inexpensive results for engineering model updating. Via demonstration on two full-scale case studies, the authors argue that error-domain model falsification is able to incorporate, iteratively and transparently, the incremental information gain, stemming from the sensing networks to facilitate updating of structural models at low additional computational cost. 
Moving to utilization of sensing for triggering control actions, Azimi and Molaei Yeznabad [3] exploit a wireless-sensor network (WSN) as a main enabler for seismic vibration mitigation. Inspired by swarm intelligence, they propose a new control method, termed swarm-based parallel control (SPC), to improve seismic performance and minimize pounding hazards. This is achieved via the WSN-enabled exchange of information among adjacent buildings at corresponding floor levels. Three-dimensional time histories are simulated under earthquake excitation, with the response mitigated via use of semi-actively controlled magnetorheological (MR) dampers. The proposed control algorithm is assessed against fuzzy logic control (FLC), as well as passive on/off methods. Results indicate that in case of failure in the control system, as well as structural damage, the proposed WSN-driven SPC method can sense damage and accordingly update the control forces in the adjacent buildings to alleviate pounding.

A key factor in successful modal identification, model updating, and control is the chosen configuration of sensors. In this special issue, Barthorpe and Worden [4] offer a review of advances in sensor placement optimization (SPO) strategies for structural health monitoring (SHM). In recent years, this has been primarily examined in the context of damage identification. This work formally defines the SPO for SHM problem, offers an overview of the current state of the art, and proceeds to identify promising emergent trends. Some of the major (still) open questions pertain to robustness to modelling uncertainty, benign measurement influences, and sensor failures. A lack of sufficient studies on practical validation of the developed sensor designs is further remarked. It is finally argued that, since such a design is carried out at an offline design stage, it is not required to devote more effort in accelerating the proposed optimization schemes.

The issue of sensing extends beyond what is deemed as traditional civil infrastructure. In their work, Al-Obaidi et al. [5] study the transport processes of solids due to geophysical flows via use of a novel sensing mechanism; a miniaturized instrumented particle that can provide a direct, non-intrusive, low-cost, and accessible method for the assessment of coarse sediment particle entrainment. The presented instrumented particle is fitted with a triaxial MEMs accelerometer, a magnetometer and angular displacement sensors, which enable monitoring of the particle's 3D motion at a configurable rate of 200-1000 Hz. An appropriate filter is used for inertial sensor fusion, uncertainty mitigation, and noise reduction. The instrumented particle is tested under controlled lab conditions, reproducing initiation of the destabilization of a bed surface in an open channel flow. The work showcases the potential of custom designed and appropriately calibrated instrumented particles in the context of monitoring the possible instigation of water infrastructure hazards.

The final contribution underlines the importance of delivering methods able to tackle the challenge of uncertainty in the environmental and operational conditions (EOCs) that a monitored system is exposed to, and more importantly, do so in absence of availability of physics-based models. In a conventional setting, varying dynamic response can be captured via use of parameter-varying extensions of the original autoregressive with exogenous input (ARX) model. Yet, EOCs tend to vary in time-scales (days, weeks, months) that are sufficiently longer to the system dynamics. Tatsis et al. [6] propose a distributed framework for structural identification, damage detection, and localization by extending the conventional ARX structure. Here, the long time-scale dependence on EOCs is captured via a Gaussian process regression, while a distributed residual generation algorithm is set up for damage detection. Finally, damage localization is demonstrated via computation of the ARX-inferred mode shape curvatures. The diagnostic capabilities of the proposed scheme under varying EOCS are verified on two simulated case studies of increasing complexity.

This special issue has proposed contributions across the complete SHM information chain; from sensor design to configuration (placement), data interpretation, and triggering of reactive action (control). The featured papers offer an overview of the state-of-the-art and further proceed to introduce novel methods and tools. Several of these particularly target the treatment of uncertainty, which inherently describes the sensed information and the behavior of monitored systems. It is hoped 
that the readers will appreciate this Special Issue enhancing their knowledge in the area and helping them advance their ongoing research and innovation activities in sensing for SHM.

Conflicts of Interest: The authors declare no conflict of interest.

\section{References}

1. Argyris, C.; Papadimitriou, C.; Panetsos, P.; Tsopelas, P. Bayesian model-updating using features of modal data: Application to the Metsovo Bridge. J. Sens. Actuator Netw. 2020, 9, 27. [CrossRef]

2. Pai, S.; Reuland, Y.; Smith, I. Data-interpretation methodologies for practical asset-management. J. Sens. Actuator Netw. 2019, 8, 36. [CrossRef]

3. Azimi, M.; Molaei Yeznabad, A. Swarm-based parallel control of adjacent irregular buildings considering soil-structure interaction. J. Sens. Actuator Netw. 2020, 9, 18. [CrossRef]

4. Barthorpe, R.; Worden, K. Emerging trends in optimal structural health monitoring system design: From sensor placement to system evaluation. J. Sens. Actuator Netw. 2020, 9, 31. [CrossRef]

5. Al-Obaidi, K.; Xu, Y.; Valyrakis, M. The design and calibration of instrumented particles for assessing water infrastructure hazards. J. Sens. Actuator Netw. 2020, 9, 36. [CrossRef]

6. Tatsis, K.; Dertimanis, V.; Ou, Y.; Chatzi, E. GP-ARX-Based structural damage detection and localization under varying environmental conditions. J. Sens. Actuator Netw. 2020, 9, 41. [CrossRef]

(C) 2020 by the authors. Licensee MDPI, Basel, Switzerland. This article is an open access article distributed under the terms and conditions of the Creative Commons Attribution (CC BY) license (http://creativecommons.org/licenses/by/4.0/). 